\title{
Hyalomma asiaticum et Hyalomma dromedarii Comparaison des larves
}

\author{
par G. SENEVET \\ (Laboratoire de Parasitologie de la Faculté de Médecine de Paris \\ 12, rue de l'Ecole-de-Médecine, Paris, $\sigma^{\mathrm{e}}-\left[\mathrm{P}^{\mathrm{r}} \mathrm{M}\right.$. LARIVIÈRE])
}

\begin{abstract}
Résumé
La synonymie entre $H$. asiaticum et $H$. dromedarii, admise par Delpy (1937), est rejetée par $\mathbf{M}^{\mathrm{me}}$ Feldman-Musham, Pomeranzev, Feider et Mironescu, Kaiser et Hoogstraal.

Senevet a recherché, par comparaison avec la description de «asiaticum» par Feider et Mironescu, s'il existait des différences entre cette larve et celle de $H$. dromedarii.

Il existe de faibles différences qui seraient peut-être insuffisantes pour justifier à elles seules la séparation des deux espèces.
\end{abstract}

\section{Summary}

Delpy (1937) put in synonymy $H$. asiaticum and $H$. dromedarii. More recent authors: Feldman-Muhsam, Pomeranzev, Feider and Mironescu, and Kaiser and Hoogstraal think those two Hyalomma are distinct species.

Senevet tried to find wether there were differences between larvae of $H$. dromedarii and the description of those of $H$. asiaticum by Feider and Mironescu.

Small differences exist, but perhaps too small to separate alone both species.

L'identité ou la dualité de ces deux espèces a été très discutée. Delpy (1937), à la suite de ses patientes études sur $H$. dromedarii (Koch, 1844), condamne formellement un certain nombre d'espèces et de sous-espèces, parmi lesquelles Hyalomma dromedarii asiaticum (Schulze et Schlottke, 1929), et $H$. asiaticum (Schulze), qu'il considère comme synonymes de $H$. dromedarii (Koch, 1844). 


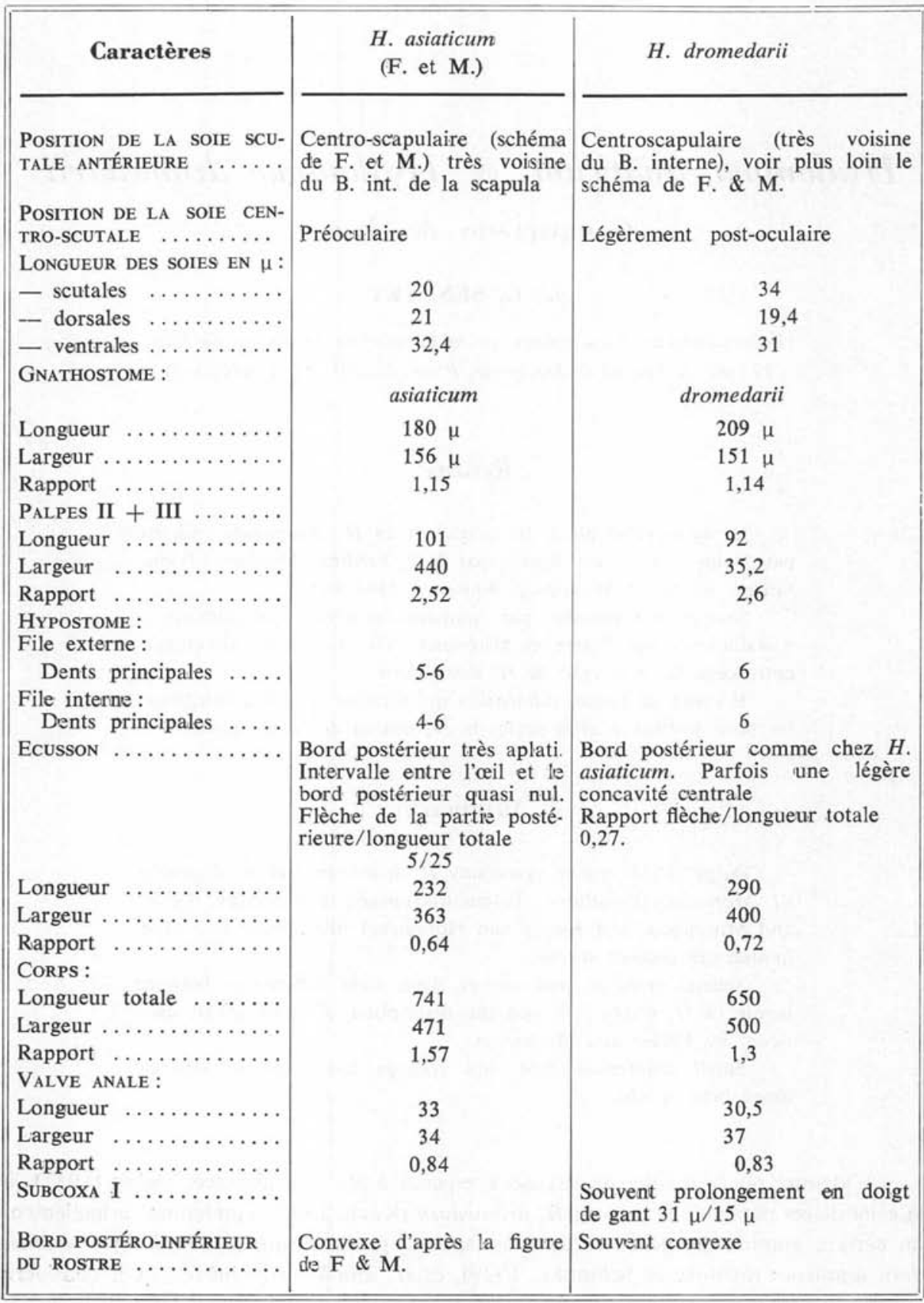

Toutes les longueurs indiquées ci-après sont exprimées en $\mu$. 
Au contraire, $\mathbf{M}^{\mathrm{me}}$ Feldman-Musham (1954) rejette de façon formelle cette synonymie (p. 135).

Pomeranzev (1950), tout en reconnaissant la proximité très grande de ces deux espèces, retient $H$. asiaticum comme autonome et en décrit le mâle et la femelle.

Feider et Mironescu, dans leur étude sur les Hyalomma de Roumanie (1961), considèrent $H$. asiaticum comme une espèce valable. Nous utiliserons plus loin leur travail.

Enfin, Kaiser et Hoogstraal (1963), à propos des tiques de l'Afghanistan, décrivent séparément $H$. dromedarii asiaticum (Schulze et Schlottke, 1929) et $H$. dromedarii (Koch, 1744).

En quasi-unanimité, les auteurs récents séparent donc les deux espèces.

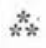

Etant donné que la majeure partie des travaux précédents n'a trait qu'aux formes adultes, j'ai cherché à voir si l'on pouvait trouver une différence entre les larves de ces deux espèces.

Je disposais dans les collections du laboratoire de parasitologie de la Faculté de Médecine de Paris de nombreuses larves de $H$. dromedarii, provenant de l'Afrique du Nord. Je vais donc, dans cette note, les comparer au schéma descriptif de Feider et Mironescu, pour les larves de $H$. asiaticum. Cette comparaison sera suivie de quelques remarques sur les larves de $H$. dromedarii.

La comparaison des deux tableaux montre que les larves de $H$. asiaticum et $H$. dromedarii sont extrêmement voisines.

Cependant, on peut, sous toutes réserves, signaler comme différences:

a) la position de la ligne des soies scutales 3 légèrement en avant de la ligne des yeux chez $H$. dromedarii au lieu d'être légèrement en arrière comme chez $H$. asiaticum ;

b) en outre, d'une manière générale, la taille du rostre est plus élevée (209 $\mu$ chez dromedarii contre $180 \mu$ chez asiaticum, mais le rapport L/la est pratiquement le même : 1,14 contre 1,19. Les palpes sont un peu plus courts chez dromedarii. Egalement pour la longueur totale, plus courte, et index plus petit. Enfin, toujours chez dromedarii, les dimensions de l'écusson sont un peu supérieures, ainsi que le rapport $\mathrm{L} /$ la.

En définitive, notre recherche n'impose pas la dualité des espèces, mais elle ne s'y oppose pas davantage. 


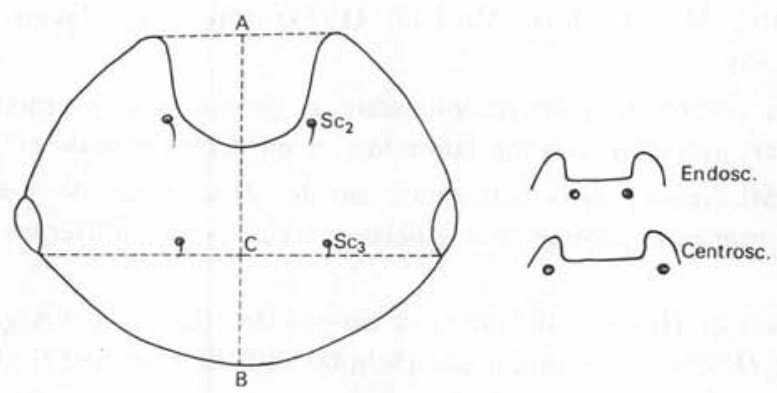

A gauche: Ecusson dorsal de la larve de $H$. dromedarii. Sc 2 : scutale antérieure, Sc 3: scutale postérieure. La ligne centrale en pointillé : $\mathrm{AB}$ : longueur totale de l'écusson, $\mathrm{CB}$ : flèche mesurant la courbure du bord postérieur de l'écusson

A droite: position de Sc 2 : endoscap.: endoscapulaire, centroscap.: centroscapulaire (d'après le schéma de Feider)

\section{Bibliographie}

Delpy (L.), 1936. - Notes sur les Ixodidés du genre Hyalomma (Koch 1844). Ann. Parasit. hum. et comp., XIV (3), p. 343.

-, 1937. - Description de Hyalomma dromedarii (Koch 1844). Morphologie de la larve et de la nymphe. Ibid., XV (6), 181-186 (nous n'avons pas utilisé cette description faite sur un autre plan et qui ne permettait pas la comparaison avec le travail de Feider et Mironescu).

Feider (L.) et Mironescu (L.), 1961. - Contributii la cunoasterea morfologici unor larve ale genului Hyalomma (Koch 1844). Annalele stiintifice ale Universitatii «Al. I; Cuza » din Iasi. Serie noua Sectiunea II (Stiinte naturale), t. VII, Fasc. 2, 305-311 (VII planches).

Feldman-Musham (B.), 1954. - Revision of the genus Hyalomma. I. Description of Koch's types. Bullet. of the Res. Council of Israel, V, IV (2), 151-170.

Kaiser (M. N.) et HoogstraAl (H.), 1963. - The Hyalomma ticks (Ixodoidea-Ixodidae) of Afghanistan. The Journ. of Parasit., XL (1), 130-139. 